much the most likely direction of assault from a lizard.' According to this writer, it is when the butterfly requires to rest that it settles, not on the under side of a leaf, as do most other butterflies, but ' on the bare trunk, or one of the larger boughs, of a tree.'

NAT. Sct.

\section{SHORTER ARTICLES}

DATA WITH a POSSIBLE BEARING ON THE CAUSE OF LIGHTNING.

1. Lenard inferred from his experiments that it is necessary for the water jet to strike a solid obstacle to generate the electricty observed in the surrounding medium of air. I find that a surface of water is also efficient, and I place the electricity as a charge on the water nuclei produced, because the charge increases with the number of nuclei computed from their coronas. In other words, the mere attrition of water by water is sufficient to charge the nuclei.

In a forthcoming paper in the American Journal of Science I show that if each nucleus carries one electron, there must be at least $10^{6}$ nuclei per cubic cm. 'At least,' because much of the charge is lost in the tube which conveys the nuclei into the condenser, and I have not yet allowed for this.

From the coronas simultaneously produced I find that about 5,000 nuclei are present per c.c. Hence each nucleus carries 200 electrons, while its potential is below five volts. Thus there are a million electrons in each c.c. of the air which I examine, or in a cubic kilometer there would be $10^{21}$ electrons, or $7 \times 10^{11}$ electrostatic units of charge, or about 200 coulombs.

2. Let this region be spherical with a superficial capacity, which would then be $.62 \times 10^{5} \mathrm{~cm}$. The potential * at the surface of the region would be eleven million electrostatic units of potential, or over three thousand million volts, if the nuclei were all of the same sign and were transferred to the surface. Every time the region is emptied of its nuclei, the surface acquires a charge of

* For a mile flash of lightning 70 coulombs at a million volts are usually conceded (Lodge). the enormous potential stated, and there is no reason why the nuclei may not be continually produced by attrition while they are being transferred.

3. Now regarding the transfer of nuclei, we may note that when they are produced from pure water, positive charges are usually in excess; when produced from dilute solutions, negative charges are usually in excess; but I find that the bulk of the nuclei are symmetrically positive and negative.

The velocity of the nucleus of charge $e$, in an electrostatic field of the potential gradient, $E$, is $v=E e / 6 \pi \mu R$, where $R$ is the radius of the nucleus and $\mu$ (.0002, say), the viscosity of air. Put, therefore, in this equation the values which $I$ have here and in other places adduced, $R=10^{-6} \mathrm{~cm}$., $e=200 \times 7 \times 10^{-10}$ electrostatic units, whence $v=37 \mathrm{~cm} . / \mathrm{sec}$, or over four fifths mile an hour, for the unit electrostatic field; about .003 mile per hour of a field of one volt/cm.

Thus there is considerable mobility in these nuclei. With a strong electrostatic field at least locally in action, the nuclei of one sign would thus be driven outward, warmer nuclei into colder regions of continually increasing conduction or rarer air, where their charges would be dissipated. The other nuclei would be driven earthward, colder nuclei into warmer regions of continually decreasing conduction to be discharged, if at all, by a flash, particularly if, on growth of drops, gravity steps in as a final motor.

Whether there is sufficient commotion in thunder-storms to give rise to the attrition of water, whether comminution will not suffice if accomplished in other ways, whether an earth electrostatic field is an adequately permanent or localized occurrence, whether indeed separation of nuclei is needed if there is enough excess of charges of definite sign, must be left for further consideration; but it seems to me that data bearing on the occurrence of lightning are here suggested which deserve serious scrutiny.

BRowN UNIVERSITY,

C. Barus.

Providence, R. I. 